\title{
A stable transcription factor complex nucleated by oligomeric AML1-ETO controls leukaemogenesis
}

\author{
Xiao-Jian Sun ${ }^{1}$, Zhanxin Wang ${ }^{2}$, Lan Wang ${ }^{3,4}$, Yanwen Jiang ${ }^{5}$, Nils Kost ${ }^{6}$, T. David Soong ${ }^{7}$, Wei-Yi Chen ${ }^{1}$, Zhanyun Tang ${ }^{1}$, \\ Tomoyoshi Nakadai ${ }^{1}$, Olivier Elemento ${ }^{7}$, Wolfgang Fischle ${ }^{6}$, Ari Melnick ${ }^{5}$, Dinshaw J. Patel ${ }^{2}$, Stephen D. Nimer ${ }^{3,4}$ \\ \& Robert G. Roeder ${ }^{1}$
}

Transcription factors are frequently altered in leukaemia through chromosomal translocation, mutation or aberrant expression ${ }^{1}$. AML1-ETO, a fusion protein generated by the $t(8 ; 21)$ translocation in acute myeloid leukaemia, is a transcription factor implicated in both gene repression and activation ${ }^{2}$. AML1-ETO oligomerization, mediated by the NHR2 domain, is critical for leukaemogenesis ${ }^{3-6}$, making it important to identify co-regulatory factors that 'read' the NHR2 oligomerization and contribute to leukaemogenesis ${ }^{4}$. Here we show that, in human leukaemic cells, AML1-ETO resides in and functions through a stable AML1-ETO-containing transcription factor complex (AETFC) that contains several haematopoietic transcription (co)factors. These AETFC components stabilize the complex through multivalent interactions, provide multiple DNAbinding domains for diverse target genes, co-localize genome wide, cooperatively regulate gene expression, and contribute to leukaemogenesis. Within the AETFC complex, AML1-ETO oligomerization is required for a specific interaction between the oligomerized NHR2 domain and a novel NHR2-binding (N2B) motif in E proteins. Crystallographic analysis of the NHR2-N2B complex reveals a unique interaction pattern in which an N2B peptide makes direct contact with side chains of two NHR2 domains as a dimer, providing a novel model of how dimeric/oligomeric transcription factors create a new protein-binding interface through dimerization/oligomerization. Intriguingly, disruption of this interaction by point mutations abrogates AML1-ETO-induced haematopoietic stem/progenitor cell self-renewal and leukaemogenesis. These results reveal new mechanisms of action of AML1-ETO, and provide a potential therapeutic target in $t(8 ; 21)$-positive acute myeloid leukaemia.

AML1-ETO consists of the DNA-binding (RUNT) domain of the haematopoietic transcription factor AML1 (also known as RUNX1) and four conserved domains (NHR1-4) of ETO (also known as RUNX1T1) ${ }^{2}$. These domains differentially contribute to AML1-ETO activities in regulating cell proliferation, differentiation and survival ${ }^{2}$. In particular, the NHR2-mediated oligomerization of AML1-ETO has been shown to be critical for leukaemogenesis ${ }^{3-6}$. Although oligomerization endows AML1-ETO with a DNA-binding preference for duplicated AML1 sites $^{7}$, it is important to explore the possibility that oligomerization might also affect cofactor recruitment and function. AML1-ETO is generally thought to act as a transcriptional repressor by recruiting corepressors (for example, NCOR and HDAC proteins) to AML1 target genes ${ }^{8-10}$, or by interacting and interfering with other transcription factors (for example, ETS family proteins, $\mathrm{C} / \mathrm{EBP} \alpha$, GATA1 and E proteins $)^{11-17}$. In relation to its functions in gene activation, AML1-ETO also can recruit the coactivators p300 (ref. 18) and PRMT1 (ref. 19). Beyond these indications of dynamic AML1-ETO interactions with diverse proteins, it has been unclear whether AML1-ETO resides in any stable multiprotein complex(es) that might endow it with new properties that lead to altered regulatory events and corresponding cellular functions.

To identify a natural AML1-ETO-containing complex in leukaemic cells, we used patient-derived Kasumi- 1 cells and an antigen-purified anti-ETO antibody that showed high specificity and affinity (Supplementary Fig. 2a, b). The absence of wild-type ETO in Kasumi-1 cells ${ }^{20}$ allowed selective isolation of AML1-ETO from derived nuclear extracts, which contained most of the AML1-ETO (Supplementary Fig. 2c). Using a high-stringency buffer to preclude weak or nonspecific interactions, we isolated the stable AETFC complex, whose components were identified by SDS-polyacrylamide gel electrophoresis (SDS-PAGE) (Fig. 1a) and mass spectrometry (Supplementary Fig. 3a), and confirmed by immunoblot (Fig. 1b). These components include the AML1-binding partner CBF- $\beta$, E proteins HEB and E2A, the haematopoietic E-box-binding transcription factor LYL1 (but not its homologue SCL; also known as TAL1), the LIM-domain protein LMO2 and its interacting partner LDB1. Although interactions between some of these factors (or their homologues) have been implicated in a related GATA1-SCL-E2A-LMO2-LDB1 complex in erythrocytes ${ }^{21}$, their connection with AML1-ETO in AML is unknown. A gel-filtration analysis indicated that they form a stable, high-molecular-weight complex (Supplementary Fig. 3b). We then used baculovirus vectors to reconstitute AETFC and to characterize the pairwise interactions within AETFC (Supplementary Fig. 3c, d). The results revealed an interaction network (Fig. 1c) in which several strong interactions link all the components one by one and probably have a major role in AETFC assembly, although some weak interactions may further stabilize the complex.

To assess potentially joint functions of the AETFC components, we performed a chromatin immunoprecipitation coupled with massively parallel DNA sequencing (ChIP-seq) analysis of AML1-ETO, HEB, E2A and LMO2 in Kasumi- 1 cells and validated some binding events by ChIP coupled with quantitative polymerase chain reaction (ChIPqPCR)-for example, on the well-established CSF1R FIRE ${ }^{22}$ and SPI1 $U R E^{23}$ enhancers. Comparison of their binding sites and enrichment scores indicated a genome-wide co-localization and a correlation of binding strengths, and suggested that the interactions between the components facilitate their binding to the genome (Fig. 1d and Supplementary Fig. 4a-d). Sequence analysis of AML1-ETO-binding regions revealed that both AML1 sites and E-boxes (recognized by $\mathrm{E}$ proteins and other basic helix-loop-helix (bHLH) transcription factors including LYL1) were overrepresented (Supplementary Fig. 4e, f), suggesting that, in addition to direct binding to DNA through the RUNT domain, AML1-ETO also binds to DNA indirectly through its interaction with the E-box-binding AETFC components.

To investigate the role of AETFC in regulating gene expression and leukaemogenesis, we performed gene knockdown in Kasumi-1 cells ${ }^{24}$ 

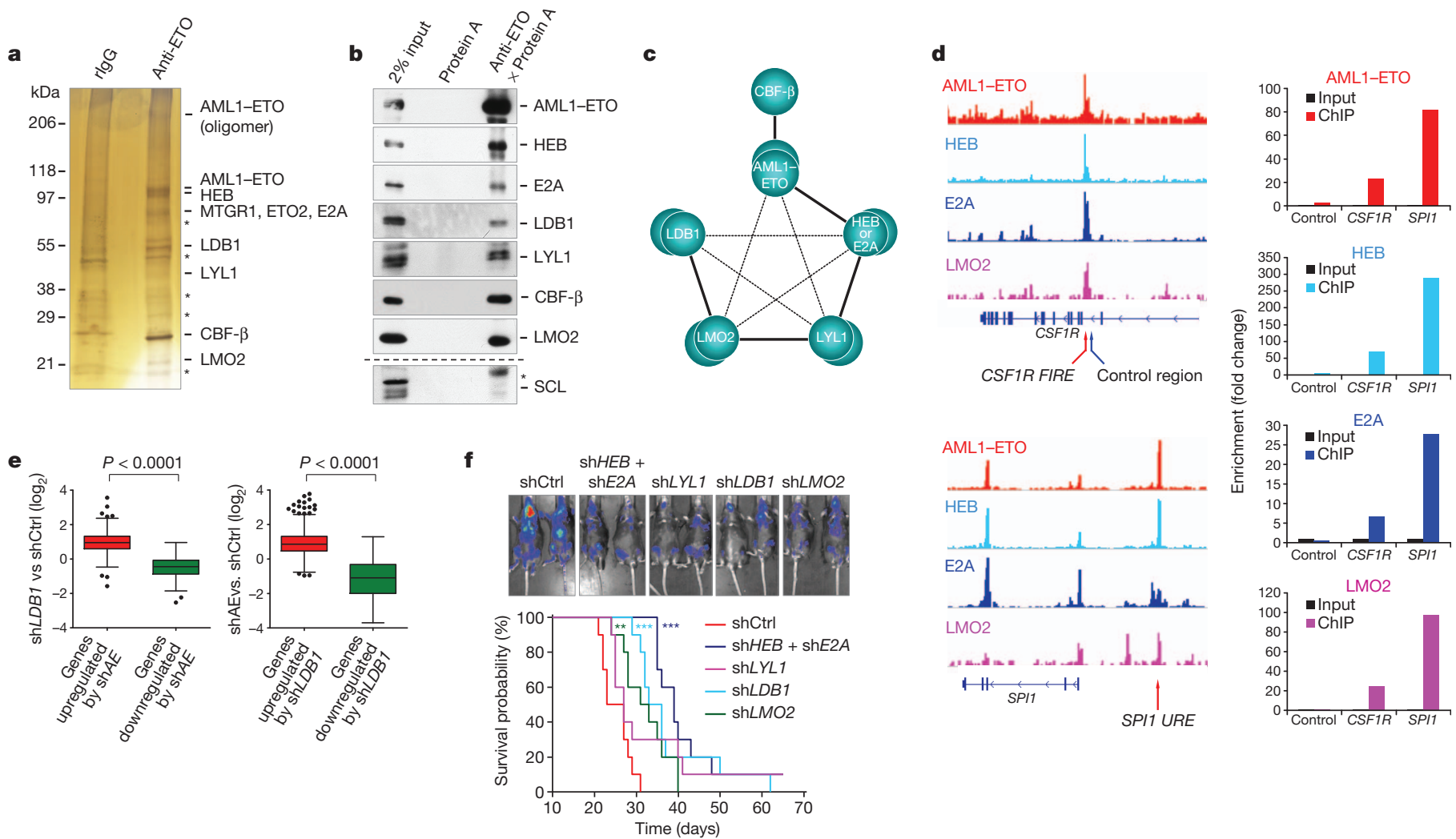

Figure $1 \mid$ AML1-ETO resides in and functions through AETFC. a, SDSPAGE and silver staining of AETFC isolated from Kasumi-1 nuclear extract. Asterisks, non-specific bands. rIgG, rabbit immunoglobulin G. b, Coimmunoprecipitation and immunoblot confirmation of AETFC components. Asterisk, IgG signal. c, Schematic of interactions within AETFC. Thick and thin lines denote strong and weak interactions, respectively. Double spheres denote

and in a mouse leukaemic model induced by AML1-ETO9a (AE9a), a leukaemogenic, truncated form of AML1-ETO ${ }^{25}$. First of all, individual knockdowns of AETFC components (but not SCL) significantly decreased some other components at the protein level but not at the messenger RNA level (Supplementary Fig. 5a, b), suggesting a mutual stabilization mechanism within AETFC. Interestingly, SCL mRNA was downregulated by knockdown of any AETFC component (Supplementary Fig. 5b). Because the $3^{\prime}$ haematopoietic enhancer of $S C L^{26}$ is bound by AETFC (Supplementary Fig. 5c), SCL is probably a direct target gene of AETFC. In an extension of this observation, global ChIP-seq and RNA-seq analyses revealed that the genes up- and downregulated by one AETFC component were similarly regulated by others (Fig. 1e and Supplementary Fig. 5d). These analyses led to the identification of a set of genes that are both directly bound and cooperatively regulated by AETFC components (Supplementary Fig. 5e and Supplementary Tables 1,2). We next showed that knockdowns of AETFC components considerably delayed leukaemogenesis in mice (Fig. If and Supplementary Fig. 6), indicating a requirement for AETFC components in AML1-ETO-mediated leukaemogenesis. Notably, double knockdown of HEB and E2A most markedly delayed leukaemogenesis, which is consistent with the primary importance of these two $\mathrm{E}$ proteins in AETFC assembly/stabilization (Fig. 1c and Supplementary Fig. 5a, f).

To investigate further the function and mechanism of these two $\mathrm{E}$ proteins in AETFC, we performed co-immunoprecipitation experiments with a series of deletion mutants of AML1-ETO and HEB, as well as a GST pull-down assay with isolated domains. These analyses clearly established multivalent interactions between AML1-ETO and E proteins and, interestingly, showed that the functionally critical NHR2 domain mediates not only oligomerization but also an interaction with E proteins (Supplementary Fig. 7; ref. 16). We next took potential homodimerization of components. d, ChIP-seq and ChIP-qPCR analyses of AETFC components on CSF1R and SPI1. e, Correlation between AE and LDB1 in regulating gene expression. shCtrl, control shRNA. f, Knockdown of AETFC components delays AE9a-induced leukaemogenesis in mice, indicated by representative bioluminescent imaging (top) and survival curves (bottom; $n=10 ; * * * P<0.001 ; * * P<0.01$ ).

several steps to clarify the relationship between NHR2 oligomerization and the NHR2-E-protein interaction. First, we found that the multisite NHR2 mutation m7, which disrupts NHR2 oligomerization ${ }^{4}$, also completely disrupts the NHR2-E-protein interaction (Fig. 2a, b). Second, we performed an exhaustive screening of NHR2 mutations and found that NHR2 oligomerization and the NHR2-E-protein interaction involve different surfaces of the NHR2 $\alpha$-helix (Fig. 2a-d). Accordingly, we identified point mutations that specifically disrupt the NHR2-E-protein interaction (Fig. 2b) but not NHR2 oligomerization, as indicated by co-immunoprecipitation (Supplementary Fig. 8) and gel-filtration (Fig. 2c) assays. Third, we found that the mutantwild-type AML1-ETO heterodimer, unlike the wild-type homodimer, failed to bind $\operatorname{HEB}(\triangle \mathrm{AD} 1)$ (Fig. 2e), indicating that a single wild-type AML1-ETO in the dimer is unable to bind $\operatorname{HEB}(\triangle \mathrm{AD} 1)$ and, thus, that NHR2 oligomerization is required for the NHR2-E-protein interaction.

To clarify further the mechanism of E-protein recognition of the NHR2 oligomer, we first mapped the N2B region in HEB. As a result, we identified a novel, 18-amino-acid N2B motif that is required for the NHR2 interaction (Fig. 3a, b and Supplementary Fig. 9). A subsequent X-ray crystallographic analysis of the NHR2-N2B complex showed that NHR2 formed a tetrameric $\alpha$-helical bundle $e^{4}$ with four N2B peptides bound on two symmetrically related surfaces with 1:1 stoichiometry (Fig. 3c and Supplementary Fig. 10a). After an independent validation of the directionality and register of the bound N2B peptide (Supplementary Fig. 10b, c), we demonstrated that a given N2B binds to a narrow surface channel created by two NHR2 helices, with P191 and S192 of N2B inserted deeply into a surface pocket (Fig. 3d). Notably, P191 of N2B interacts through hydrogen bonding with S522 from one NHR2 helix, while S192 and V194 form hydrogen bonds with E501 and H504, respectively, from another NHR2 helix 
a Hs MTGR1

$M m \_M T G R 1$

Dr_MTGR1

Mm_ETO

Hs ETO

Dr ETO

Hs_ETO2

Mm_ETO2

Dr_ETO2

Dm_Nvy

\section{DELVDHR}

QDELVDHR

REEPVDHR

TEREWAEEWRHLDHVI

OEEMIDHRLTDREWAEEWKHLDHLI

QEEVIDHRLTDREWAEEWKHLD HLL

QEEVIDHKLTEREWAEEWKHLNNLLN

QEEVIDHRLTEREWAEEWKHLNSLLN

QEEVIDHRLTDREWAEEWKHLDNLL

NPNAAAP GAP GAGGEEEWKNIHTMLNC
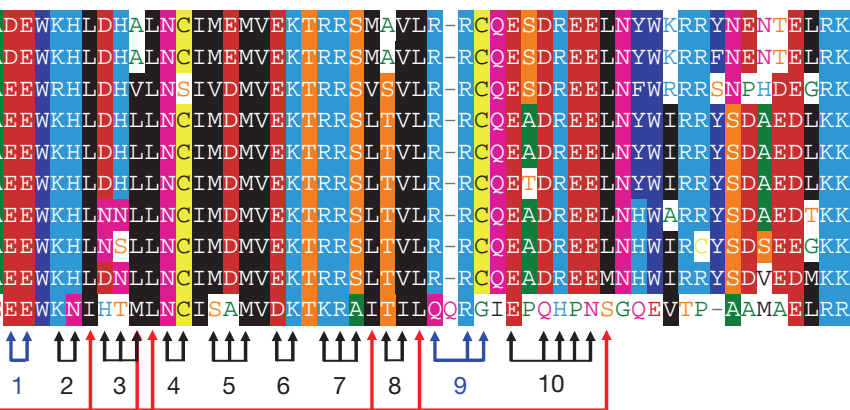

45

f:HEB( $\triangle A D 1)$

b

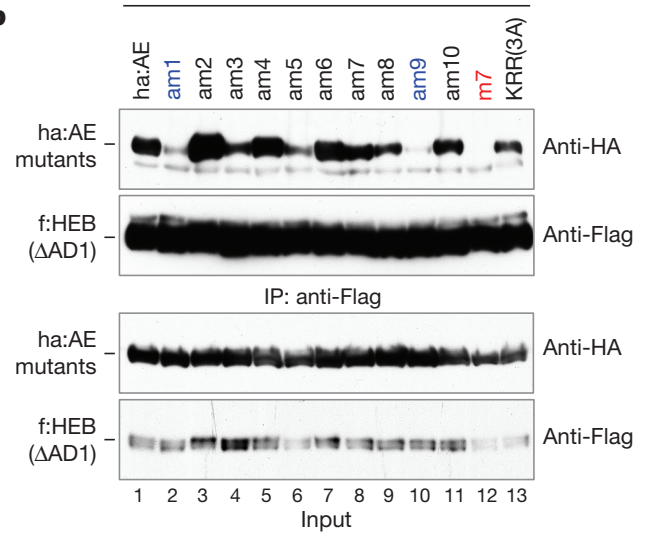

$\mathrm{m} 7$

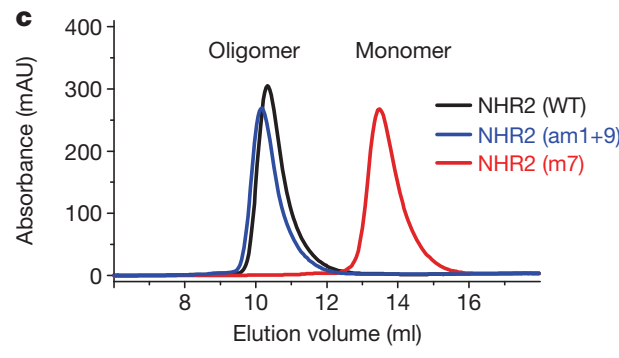

d

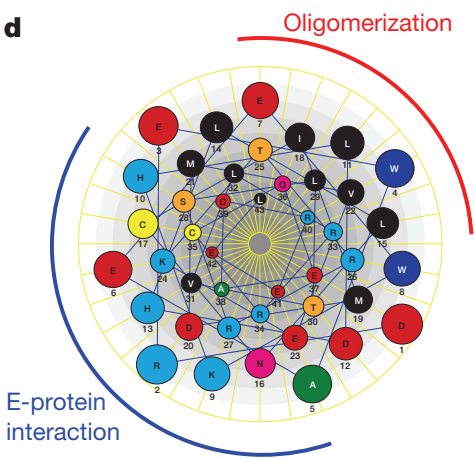

e

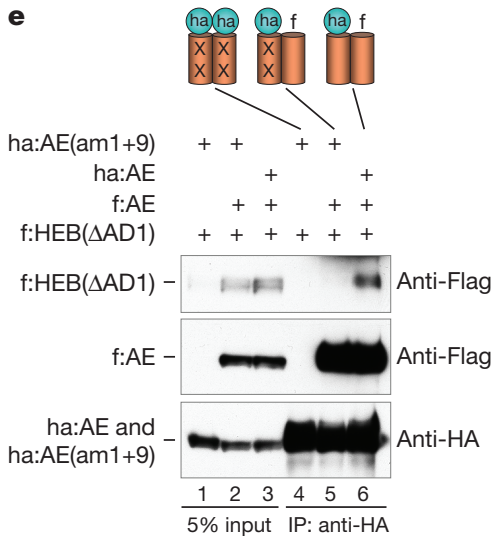

Figure $2 \mid$ The oligomerized AML1-ETO NHR2 domain mediates a specific interaction with E proteins. a, Alignment of the NHR2 of ETO family proteins. Arrows denote residues mutated to alanine in mutants am1-am10, which were screened in this study, and the $\mathrm{m} 7$ mutant that disrupts NHR2 oligomerization ${ }^{4}$. Hs, human; Mm, mouse; Dr, zebrafish; Dm, fruit fly. b. Identification of mutations that disrupt the interaction between $\mathrm{AE}$ and $\mathrm{HEB}(\triangle \mathrm{AD} 1)$. f, Flag tag; ha, HA tag. c, Gel-filtration profiles of wild-type (WT) and mutant NHR2. Wild-type NHR2 and am1 +9 NHR2 were eluted as oligomers, and the m7 mutant was eluted as a monomer. d, Distinct surfaces of the NHR2 $\alpha$-helix mediate oligomerization and the E-protein interaction. e, NHR2 oligomerization is required for the NHR2-E-protein interaction. Mutantmutant-AE homodimer (lanes 1,4), mutant-wild-type heterodimer (lanes 2, 5) and wild-type-wild-type homodimer (lanes 3,6) were formed in 293T cells by co-expression of wild-type AE and NHR2-mutated AE (XX) that fails to interact with $\mathrm{HEB}(\triangle \mathrm{AD} 1)$, and their abilities to bind to $\mathrm{HEB}(\triangle \mathrm{AD} 1)$ were determined by co-immunoprecipitation. IP, immunoprecipitant. XI_MGC52608
XI_tcfe2a
Gg_TCF3
Hs_E2A
Mm_E2A
Mm_HEB
Gg_HTF4
Hs_HEB
XI_MGC_83361
Gg_TCF12
Hs_E2-2
Mm_E2-2
Dr_tcf12
Dr_zgc_113045
Dr_Tcf3

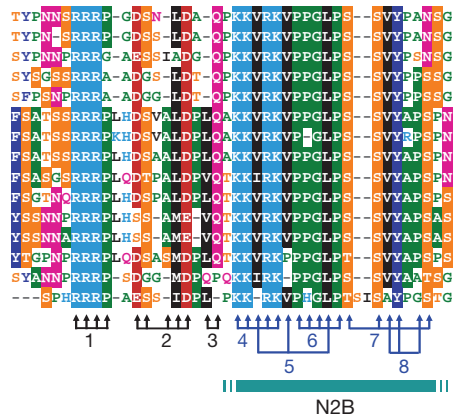

c

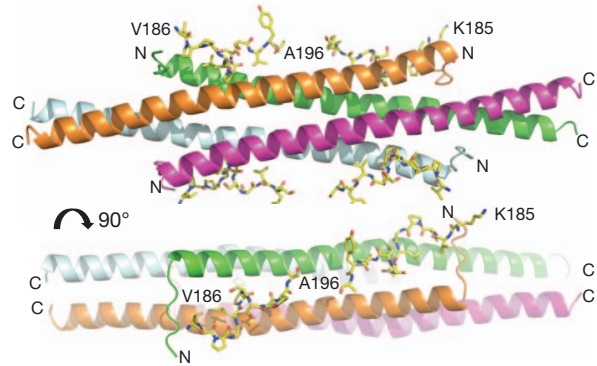

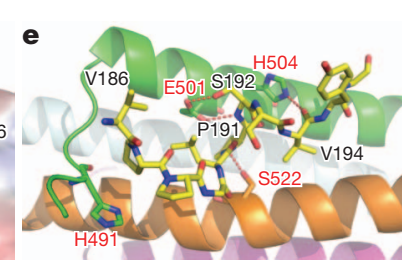

b

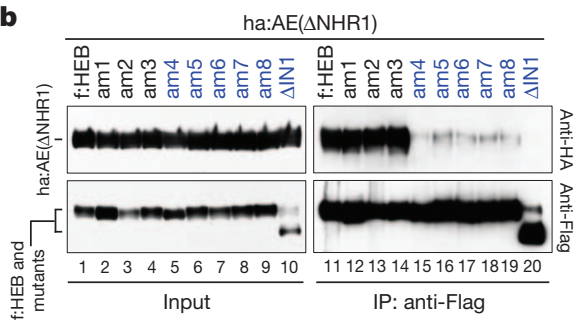

$\mathbf{f}$

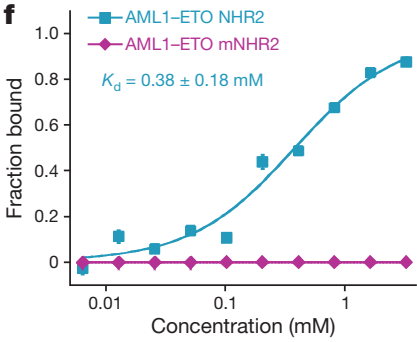

g

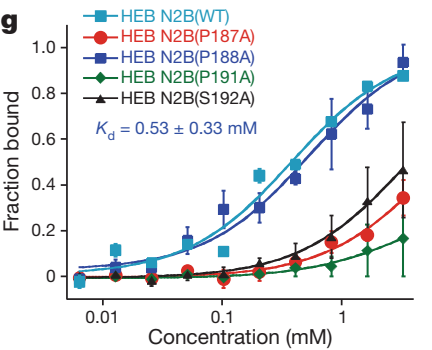

Figure $3 \mid$ Structural details of the NHR2-N2B interaction. a, Alignment of the N2B regions of vertebrate $E$ proteins. Arrows denote residues mutated to alanine in mutants am1-am8. Gg, chicken; Xl, frog. b, Identification of the N2B motif by co-immunoprecipitation. c, Side (top) and look-down (bottom) ribbon views of the structure of the complex between the $\mathrm{N} 2 \mathrm{~B}(177-200)$ peptide and the tetrameric-helical bundle of NHR2. The N2B peptide is shown in stick representation, and the NHR2 helices in ribbon representation. d, Positioning of the N2B peptide on the electrostatic surface of the NHR2 tetramer. e, One N2B peptide interacts with two NHR2 helices through hydrogen bonds. f, $\mathbf{g}$, Fluorescence-polarization analysis of the binding between NHR2 and a shorter N2B(182-196) peptide, comparing wild-type (WT) and indicated mutants of NHR2 (f) and $\mathrm{N} 2 \mathrm{~B}(\mathrm{~g}) . n=3$; mean \pm standard deviation (s.d.).

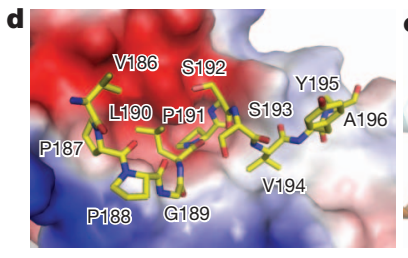


(Fig. 3e). This unique interaction pattern confirms the results of the cell-based assay (Fig. 2e) by showing, unequivocally, that two dimerized NHR2 helices are required for the N2B interaction. To verify the binding specificity further, we made mutations in NHR2 and in a shorter $\mathrm{N} 2 \mathrm{~B}(182-196)$ peptide, and determined their interactions using fluorescence polarization assays. The results showed that the binding of such a short N2B peptide to NHR2 is relatively weak but highly specific, as the mutation in NHR2 (mNHR2; Supplementary Fig. 11a) completely abolished the binding (Fig. 3f). Reciprocally, single mutations of the key residues P187, P191 or S192 of N2B also significantly reduced the binding, whereas mutation of the less important P188 residue had very little effect (Fig. $3 \mathrm{~g}$ ).

To study the biological relevance of the AML1-ETO-E-protein interactions in leukaemogenesis, we introduced the mNHR2 mutation that specifically disrupts the NHR2-N2B interaction without affecting NHR2 oligomerization (Figs 2c, 3f and Supplementary Fig. 11a, b), as well as the mNHR1 mutation that blocks the NHR1-AD1 interaction ${ }^{27}$. Effects of the NHR1 and NHR2 mutations on AML1-ETO function were initially analysed with the human $\mathrm{CD}_{3} 4^{+}$haematopoietic stem/ progenitor cell (HSPC) self-renewal assay ${ }^{28}$. Although AML1-ETO and the mNHR1, mNHR2 and mNHR1 +2 mutants exhibited comparable expression levels in transduced CD $34^{+}$cells (Supplementary Fig. 11c), mNHR2 significantly impaired the ability of AML1-ETO to maintain $\mathrm{CD} 4^{+}$cells (Fig. 4a). We also measured the frequencies of functional HSPCs using cobblestone-area-forming cell (CAFC) and long-term culture-initiating cell (LTC-IC) assays. Notably, mNHR2 significantly reduced the CAFC and LTC-IC colonies in both assays, whereas mNHR1 showed relatively mild effects and no clear synergy between mNHR1 and mNHR2 was evident (Fig. 4b, c). These results suggest that the NHR2-N2B interaction, rather than simply acting additively with the NHR1-AD1 interaction to increase binding affinity, may act as a conformational switch within AETFC (Supplementary Fig. 1a) and thus contribute to AML1-ETO-enhanced HSPC self-renewal. By contrast, these mutations showed no effect on AML1-ETO activities in differentiation inhibition (Supplementary Fig. 12) and cell growth arrest (data not shown). To exclude the possibility that mNHR2 may affect interactions other than the E-protein interaction, we designed (based on the structure) another NHR2 mutation (mNHR2S) that effected a similar loss of E-protein binding but differed completely in sequence from mNHR2. Nevertheless, mNHR2 and mNHR2S showed similar effects in HSPC self-renewal and differentiation (Supplementary Fig. 13), indicating that these effects were specifically caused by disruption of the NHR2-N2B interaction.

To determine whether these interactions are also required for leukaemogenesis in vivo, we used the mouse leukaemic model ${ }^{25}$ involving transplantation of mouse HSPCs transduced with wild-type and mutated AE9a. In this assay, mice carrying AE9a-mNHR2 showed a significant delay in leukaemogenesis compared with AE9a mice. First, AE9a-mNHR2 mice showed a significantly lower white-blood-cell count than AE9a mice (Fig. 4d), consistent with peripheral blood morphology (Supplementary Fig. 14a). Second, mNHR2 mice had a lower percentage of $\mathrm{c}-\mathrm{Kit}^{+} / \mathrm{Mac}^{-}{ }^{-} / \mathrm{GFP}^{+}$leukaemic blast cells in their peripheral blood (Supplementary Fig. 14b, c). Third, undifferentiated leukaemic cells were enriched in AE9a mice but not in mNHR2 mice (Fig. 4e). Last, mNHR2 mice survived significantly longer than AE9a mice (Fig. 4f). The mNHR1 mutation again showed relatively mild effects in suppressing leukaemogenesis in mice. Taken together, both human HSPC and mouse transplantation assays clearly showed that the NHR2-N2B interaction is required for AML1-ETOmediated leukaemogenesis. Given that the extremely high stability of the NHR2 oligomer makes its therapeutic targeting very challenging ${ }^{4}$, the NHR2-N2B interaction potentially offers a new target.

In contrast to previously reported dynamic interactions of various proteins with AML1-ETO under overexpression conditions, this study provides a direct and unbiased analysis of natural AML1-ETO in leukaemic cells, and identifies an endogenous stable complex (AETFC). The
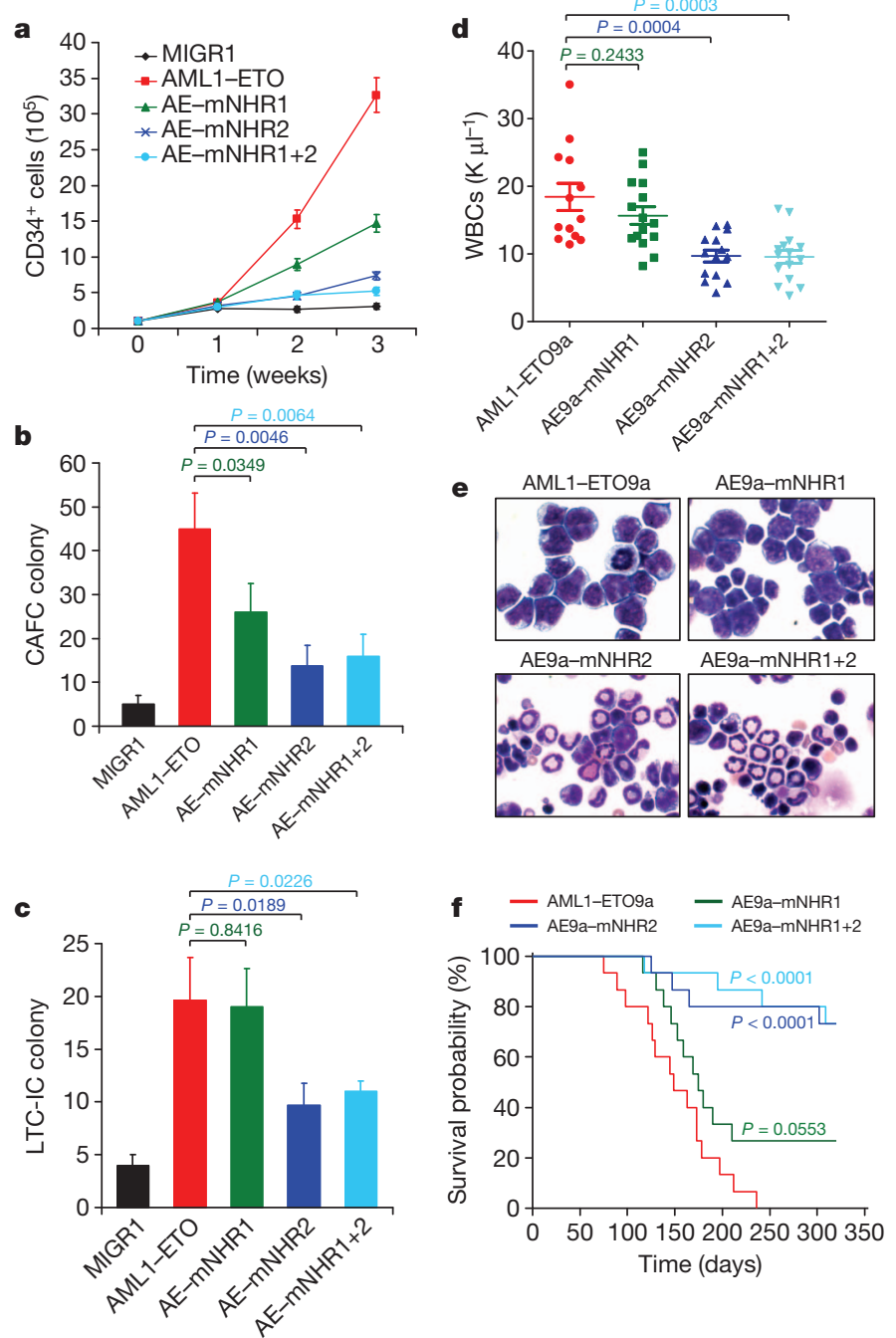

Figure 4 | Role of the AML1-ETO-E-protein interactions in human HSPC self-renewal and mouse leukaemogenesis. a, Effects of $\mathrm{AE}$ and derived mutants in the maintenance of human $\mathrm{CD} 34^{+}$cells. b, CAFC assays measuring the frequency of functional HSPCs in cells transduced with AE or derived mutants. c, LTC-IC assays measuring the frequency of functional HSPCs after long-term in vitro culture of cells transduced with $\mathrm{AE}$ or derived mutants. a-c, $n=3$; mean \pm s.d. d, Effects of AE9a and derived mutants in mouse leukaemogenesis, as indicated by white blood cell (WBC) count of peripheral blood at 4 months after transplantation. e, Morphological analysis of mouse bone marrow cells. Original magnification, $\times 1,000$. f, Kaplan-Meier survival curves of the mice $(n=15)$.

multiple components in AETFC offer opportunities for the recruitment of AML1-ETO to a variety of target genes and for the regulation of gene expression through context-dependent functional interactions with diverse transcriptional coactivators and corepressors (Supplementary Figs 1, 15; further discussion in Supplementary Information). In leukaemic cells, most, if not all, AML1-ETO probably resides in and functions through AETFC, because (1) knockdown of other AETFC components destabilizes AML1-ETO, (2) many AML1-ETO-regulated genes are likewise co-occupied and co-regulated by AETFC components, and (3) knockdowns of AETFC components significantly impair leukaemogenesis. Consistent with the observation that HEB and E2A have essential roles in AETFC assembly and stabilization, and in leukaemogenesis, the direct AML1-ETO-E-protein interaction is shown to be required for leukaemogenesis. Among the two defined AML1-ETO-E-protein interaction sites, surprisingly, the NHR2-N2B interaction, which requires oligomerization of NHR2, is necessary and sufficient for AML1-ETOmediated leukaemogenesis (further discussed in Supplementary Information). Oligomerization of transcription factors has been known to be 
an important regulatory mechanism for modulating their DNA-binding specificity and affinity; and a choice of different oligomerizing partners also contributes to the selective recruitment of cofactors ${ }^{29,30}$. The unique NHR2-N2B interaction provides a novel model in which oligomeric transcription factors use their oligomerization domains to create a new protein-binding interface. Thus, this study reveals new mechanisms of action of oligomerized AML1-ETO in leukaemogenesis, and provides potential therapeutic possibilities.

\section{METHODS SUMMARY}

Kasumi-1 cells were grown in 12-litre spinner flasks (BellCo) and the nuclear extract was prepared with our standard high-salt extraction method. Complex purification was performed in a high stringency buffer (containing $300 \mathrm{mM} \mathrm{KCl}$ and $0.1 \%$ NP-40). The Bac-to-Bac Baculovirus Expression Systems (Invitrogen) was used to reconstitute the complex in Sf9 cells. ChIP was performed with a dual cross-linking method, and ChIP- and RNA-seq were performed following Illumina protocols. The short hairpin RNA (shRNA) targeting the AML1-ETO fusion site was designed as previously described ${ }^{24}$; and other lentiviral shRNA sets were purchased from Open Biosystems. The co-immunoprecipitation assay was performed in a buffer containing $300 \mathrm{mM} \mathrm{NaCl}$ and $1 \%$ Triton X-100. Purified N2B peptide and NHR2 domain were mixed at a 1:1 ratio and crystals of the complex were grown by the hanging-drop method. Human HSPC self-renewal and differentiation assays ${ }^{18,28}$, and mouse leukaemogenesis assays ${ }^{25}$, were performed as previously described.

Full Methods and any associated references are available in the online version of the paper.

Received 17 December 2012; accepted 13 May 2013. Published online 30 June 2013.

1. Look, A. T. Oncogenic transcription factors in the human acute leukemias. Science 278, 1059-1064 (1997).

2. Peterson, L. F. \& Zhang, D. E. The 8;21 translocation in leukemogenesis. Oncogene 23, 4255-4262 (2004)

3. Minucci, S. et al. Oligomerization of RAR and AML1 transcription factors as a novel mechanism of oncogenic activation. Mol. Cell 5, 811-820 (2000).

4. Liu, Y. et al. The tetramer structure of the Nervy homology two domain, NHR2, is critical for AML1/ETO's activity. Cancer Cell 9, 249-260 (2006).

5. Kwok, C., Zeisig, B. B., Qiu, J., Dong, S. \& So, C. W. Transforming activity of AML1ETO is independent of $C B F \beta$ and ETO interaction but requires formation of homooligomeric complexes. Proc. Natl Acad. Sci. USA 106, 2853-2858 (2009).

6. Yan, M., Ahn, E. Y., Hiebert, S. W. \& Zhang, D. E. RUNX1/AML1 DNA-binding domain and ETO/MTG8 NHR2-dimerization domain are critical to AML1-ETO9a leukemogenesis. Blood 113, 883-886 (2009).

7. Okumura, A. J., Peterson, L. F., Okumura, F., Boyapati, A. \& Zhang, D. E. $\mathrm{t}(8 ; 21)(\mathrm{q} 22 ; \mathrm{q} 22)$ fusion proteins preferentially bind to duplicated AML1/RUNX1 DNA-binding sequences to differentially regulate gene expression. Blood $\mathbf{1 1 2}$, 1392-1401 (2008)

8. Gelmetti, V.etal. Aberrant recruitment of the nuclear receptor corepressor-histone deacetylase complex by the acute myeloid leukemia fusion partner ETO. Mol. Cell. Biol. 18, 7185-7191 (1998).

9. Lutterbach, B. et al. ETO, a target of $t(8 ; 21)$ in acute leukemia, interacts with the $\mathrm{N}-\mathrm{CoR}$ and $\mathrm{mSin} 3$ corepressors. Mol. Cell. Biol. 18, 7176-7184 (1998).

10. Wang, J., Hoshino, T., Redner, R. L., Kajigaya, S. \& Liu, J. M. ETO, fusion partner in $\mathrm{t}(8 ; 21)$ acute myeloid leukemia, represses transcription by interaction with the human N-CoR/mSin3/HDAC1 complex. Proc. Natl Acad. Sci. USA 95, 10860-10865 (1998)

11. Westendorf, J. J. et al. The t(8;21) fusion product, AML-1-ETO, associates with C/EBP- $\alpha$, inhibits C/EBP- $\alpha$-dependent transcription, and blocks granulocytic differentiation. Mol. Cell. Biol. 18, 322-333 (1998).

12. Mao, S., Frank, R. C., Zhang, J., Miyazaki, Y. \& Nimer, S. D. Functional and physica interactions between AML1 proteins and an ETS protein, MEF: implications for the pathogenesis of t(8;21)-positive leukemias. Mol. Cell. Biol. 19, 3635-3644 (1999).
13. Elagib, K. E. et al. RUNX1 and GATA-1 coexpression and cooperation in megakaryocytic differentiation. Blood 101, 4333-4341 (2003).

14. Zhang, J., Kalkum, M. Yamamura, S. Chait, B. T. \& Roeder, R. G. E protein silencing by the leukemogenic AML1-ETO fusion protein. Science 305, 1286-1289 (2004).

15. Gardini, A. et al. AML1/ETO oncoprotein is directed to AML1 binding regions and co-localizes with AML1 and HEB on its targets. PLoS Genet. 4, e1000275 (2008).

16. Guo, C., Hu, Q., Yan, C. \& Zhang, J. Multivalent binding of the ETO corepressor to E proteins facilitates dual repression controls targeting chromatin and the basal transcription machinery. Mol. Cell. Biol. 29, 2644-2657 (2009).

17. Martens, J. H. et al. ERG and FLI1 binding sites demarcate targets for aberrant epigenetic regulation by AML1-ETO in acute myeloid leukemia. Blood 120, 4038-4048 (2012)

18. Wang, L. et al. The leukemogenicity of AML1-ETO is dependent on site-specific lysine acetylation. Science 333, 765-769 (2011).

19. Shia, W. J. et al. PRMT1 interacts with AML1-ETO to promote its transcriptional activation and progenitor cell proliferative potential. Blood 119, 4953-4962 (2012).

20. Miyoshi, $\mathrm{H}$ et al. The $\mathrm{t}(8 \cdot 21)$ translocation in acute myeloid leukemia results in production of an AML1-MTG8 fusion transcript. EMBO J. 12, 2715-2721 (1993).

21. Wadman, I. A. et al. The LIM-only protein Lmo2 is a bridging molecule assembling an erythroid, DNA-binding complex which includes the TAL1, E47, GATA-1 and Ldb1/NLI proteins. EMBO J. 16, 3145-3157 (1997).

22. Follows, G. A. et al. Epigenetic consequences of AML1-ETO action at the human c-FMS locus. EMBO J. 22, 2798-2809 (2003).

23. Huang, G. et al. PU.1 is a major downstream target of AML1 (RUNX1) in adult mouse hematopoiesis. Nature Genet. 40, 51-60 (2008).

24. Heidenreich, O. et al. AML1/MTG8 oncogene suppression by small interfering RNAs supports myeloid differentiation of $\mathrm{t}(8 ; 21)$-positive leukemic cells. Blood 101, 3157-3163 (2003).

25. Yan, M. et al. A previously unidentified alternatively spliced isoform of $t(8 ; 21)$ transcript promotes leukemogenesis. Nature Med. 12, 945-949 (2006).

26. Sanchez, M. et al. An SCL 3' enhancer targets developing endothelium together with embryonic and adult haematopoietic progenitors. Development $\mathbf{1 2 6}$ 3891-3904 (1999).

27. Park, S. et al. Structure of the AML1-ETO eTAFH domain-HEB peptide complex and its contribution to AML1-ETO activity. Blood 113,3558-3567 (2009).

28. Mulloy, J. C. et al. The AML1-ETO fusion protein promotes the expansion of human hematopoietic stem cells. Blood 99, 15-23 (2002)

29. Amoutzias, G. D., Robertson, D. L,, Van de,P.Y.\& Oliver, S. G. Choose your partners: dimerization in eukaryotic transcription factors. Trends Biochem. Sci. 33, 220-229 (2008)

30. Funnell, A. P. \& Crossley, M. Homo- and heterodimerization in transcriptional regulation. Adv. Exp. Med. Biol. 747, 105-121 (2012).

Supplementary Information is available in the online version of the paper.

Acknowledgements We thank N. A. Speckand J. H. Bushweller for providing the AML1ETO $\mathrm{m} 7$ plasmid, and R. Baer for providing anti-SCL antibodies. This work was supported by National Institutes of Health (NIH) grants CA163086 (R.G.R.), CA129325 (R.G.R.), CA113872 (R.G.R.) and CA166835 (S.D.N.), Starr Cancer Consortium grant I5-A554 (R.G.R., D.J.P. and S.D.N.), Leukemia and Lymphoma Society (LLS) SCOR grants 7013-02 (R.G.R. and S.D.N.) and 7132-08 (R.G.R., A.M. and D.J.P.), and Rockefeller University Center for Clinical and Translational Science Pilot Project grant UL1RR024143 from NIH (X.-J.S.).X.-J.S. was a Starr Cancer Consortium Visiting Fellow. L.W. was an Empire State Stem Cell Scholar and an LLS Fellow. Y.J. was an American Society of Haematology Scholar. W.-Y.C. was an LLS Fellow. D.J.P. was supported by funds from the Abby Rockefeller Mauze Trust and the Maloris Foundation.

Author Contributions X.-J.S. and R.G.R. conceived the project. R.G.R. supervised the biochemical studies. S.D.N. supervised the leukaemia pathological studies. D.J.P. supervised the structural studies. A.M. supervised the genomic studies. X.-J.S., Z.W. L.W., Y.J., N.K., T.D.S., W.-Y.C., Z.T., T.N., O.E. and W.F. performed the experiments and analysed the data. X.J.S. and R.G.R. wrote the paper.

Author Information ChIP-seq and RNA-seq data have been deposited in the Gene Expression Omnibus under accession GSE43834. The crystal structure of the NHR2 N2B complex has been deposited in the Protein Data Bank under accession 4JOL. Reprints and permissions information is available at www.nature.com/reprints. The authors declare no competing financial interests. Readers are welcome to comment on the online version of the paper. Correspondence and requests for materials should be addressed to R.G.R. (roeder@rockefeller.edu). 


\section{METHODS}

Protein purification and mass spectrometry. Kasumi- 1 cells were grown in 12 litre spinner flasks (BellCo) and the nuclear extract was prepared by our standard high-salt extraction method ${ }^{31}$. A custom anti-ETO antibody (Covance) was developed against a C-terminal peptide of ETO and purified with a recombinant protein. Complex purification was performed in buffer BC300 $(20 \mathrm{mM}$ Tris, $\mathrm{pH}$ $7.3,300 \mathrm{mM} \mathrm{KCl}, 0.2 \mathrm{mM}$ EDTA and $20 \%$ glycerol) plus $0.1 \%$ NP-40. Eluted proteins were separated by SDS-PAGE and visible bands on the gel were sliced separately and subjected to liquid chromatography coupled with tandem mass spectrometry (LC-MS/MS) analysis. For immunoblots, the following antibodies were used: ETO (Santa Cruz; sc-9737), HEB (Santa Cruz; sc-357), E2A (Santa Cruz; sc-763), LDB1 (Santa Cruz; sc-11198), LYL1 (Abcam; ab30334), CBF- $\beta$ (Pierce; PA1-317), LMO2 (Abcam; ab81988) and SCL (a gift from R. Baer).

Gel filtration. The antigen-eluted complex was subjected to gel filtration with a Precision Column PC 3.2/30 that was pre-packed with Superose 6 and connected to an AKTA FPLC system (GE Healthcare). A Superdex 75 column (GE Healthcare) was used to determine the oligomeric states of wild-type and mutant AML1-ETO NHR2.

Reconstitution of protein complex. The Bac-to-Bac Baculovirus Expression Systems (Invitrogen) was used to generate recombinant baculoviruses containing each AETFC component. Reconstituted protein complexes were purified from Sf 9 insect cells co-infected with different baculoviruses.

ChIP and ChIP-seq. Antigen-purified anti-ETO antibody and antibodies against HEB (Santa Cruz; sc-357), E2A (Santa Cruz; sc-763) and LMO2 (Abcam; ab81988) were used for ChIP and ChIP-seq assays. ChIP was performed with a dual crosslinking method. ChIP-seq libraries were constructed following the Illumina protocol and sequenced on the Illumina Genome Analyzer II. Peak-calling/annotation and consensus sequence analyses were performed with ChIPseeqer ${ }^{32}$, CisFinder $^{33}$ and Cisgenome ${ }^{34}$.

shRNA knockdown. The shRNA against AML1-ETO was designed to target the fusion site ${ }^{24}$. Other lentiviral shRNA sets were purchased from Open Biosystems. Virus preparation and cell infection were performed according to the manufacturer's protocol. Knockdown efficiencies were analysed by RT-qPCR and/or immunoblot at 4 days after infection.

RNA-seq. Kasumi- 1 cells with knockdowns of AETFC components were harvested at 4 days after infection and RNA was extracted with TRIzol Reagent (Invitrogen). Libraries were generated using the TruSeq RNA Sample Preparation Kit (Illumina) and sequenced with Illumina HiSeq2000. The reads were aligned with TopHat and then processed with an in-house program to calculate the reads per kilobase per million reads (RPKM) value for each gene following published methods ${ }^{35}$.

Cell transfection and co-immunoprecipitation. 293T cells were transfected using Lipofactamine 2000 (Invitrogen). Cell lysis and co-immunoprecipitation were performed with T/G lysis buffer $(20 \mathrm{mM}$ Tris- $\mathrm{HCl}, \mathrm{pH} 7.5,300 \mathrm{mM} \mathrm{NaCl}$, $50 \mathrm{mM} \mathrm{NaF}, 2 \mathrm{mM}$ EDTA, $1 \%$ Triton X-100 and 20\% glycerol), and proteins were analysed by immunoblot.

GST pull-down. GST-tagged proteins were expressed in Escherichia coli and purified with Glutathione Sepharose 4B (GE Healthcare). The ${ }^{35} \mathrm{~S}$-labelled prey proteins were synthesized with the TNT Quick Coupled Transcription/Translation System (Promega). Pull-down assays were performed with NETN buffer (20 mM Tris- $\mathrm{HCl}, \mathrm{pH}$ 8.0, $100 \mathrm{mM} \mathrm{NaCl}, 1 \mathrm{mM}$ EDTA and 0.5\% NP-40), followed by SDS-PAGE and autoradiography analyses.

Co-expression of N2B fragment and NHR2 domain. The His-Sumo-tagged N2B(100-219) fragment of HEB and the GST-tagged NHR2(482-551) domain of AML1-ETO were co-expressed in E. coli and purified by GST or His pull-down. By serial truncation of N2B from both ends, a short N2B(177-200) peptide was shown to retain its binding affinity for NHR2, and this N2B fragment was used for crystallization trials.

Crystallization and structure determination. Purified N2B(177-200) peptide and NHR2(486-548) domain were mixed at around 1:1 ratio and subjected to crystallization trials. Crystals of the complex were grown by the hanging-drop method at $20^{\circ} \mathrm{C}$ in buffer containing $0.1 \mathrm{mM}$ Tris (pH 8.5) and $20 \%$ ethanol. A $2.9 \AA$ resolution data set of the complex was collected on beamline 24ID-E at the Advanced Photon Source, Argonne National Laboratory. The initial model was solved by molecular replacement using the free NHR2 structure as the search model. The validation of the directionality and register of the bound N2B peptide were performed with the selenomethionine-labelling method following the introduction of V186M, V194M and corresponding double mutations.

Fluorescence-polarization-based measurements. Peptides were labelled with fluorescein at the $\mathrm{N}$ terminus. Fluorescence-polarization assays were carried out and analysed using FP buffer $(10 \mathrm{mM} \text { TEA, } 20 \mathrm{mM} \mathrm{NaCl}, \mathrm{pH} 7.4)^{36}$. Titration series of protein in $10 \mu \mathrm{l}$ volume and containing $100 \mathrm{nM}$ fluorescinated peptide in 384-well plates were read multiple times on a Plate Chameleon II plate reader (HIDEX Oy).

Human HSPC self-renewal and differentiation. $\mathrm{CD} 34^{+}$cells were purified from human cord blood samples using the MACS CD34 Isolation Kit (Miltenyi Biotec). After expansion, cells were infected with MIGR1-derived retroviruses. GFP ${ }^{+}$ $\mathrm{CD}_{3}{ }^{+}$cells were sorted and grown in basic or differentiation medium and replated weekly. Numbers of HSPC and differentiated cells were determined by cell counting and flow cytometry. For the CAFC assays, CD34 ${ }^{+}$cells were co-cultured on an MS-5 monolayer and demi-depopulated weekly. The cobblestone areas were scored at week 5. For LTC-IC assays, the cells were plated at week 6 in methylcellulose with cytokines, and the colony numbers were scored 14 days after seeding ${ }^{18,28}$ Mouse fetal liver transplantation and leukaemogenesis assays. Mouse fetal liver HSPCs were infected with MIGR1-derived retroviruses and cultured in X-VIVO medium (Lonza) with cytokines. Recipient mice were lethally irradiated and transduced fetal liver cells were transplanted into recipient mice by tail-vein injection. Leukaemia development was determined by complete blood count, flow cytometry and morphological analyses of peripheral blood and bone marrow cells ${ }^{25}$. The overall survival of the mice was analysed by the Kaplan-Meier method, and the statistical significance was evaluated by the logrank test. For bioluminescent imaging of leukaemia in mice ${ }^{37}$, AE9a leukaemic cells were transduced with MSCV-Luciferase PGK-hygo (Addgene). On injection of the substrate of luciferase, D-Luciferin, bioluminescent imaging was performed using an IVIS100 imaging system. For gene knockdown assays in the mouse model, a mouse leukaemic cell line was generated from the spleen of the mice bearing AE9a-induced leukaemia. These cells were infected with shRNA lentiviruses, selected by puromycin and injected into recipient mice to generate leukaemia.

31. Dignam, J. D., Lebovitz, R. M. \& Roeder, R. G. Accurate transcription initiation by RNA polymerase II in a soluble extract from isolated mammalian nuclei. Nucleic Acids Res. 11, 1475-1489 (1983)

32. Giannopoulou, E. G. \& Elemento, O. An integrated ChIP-seq analysis platform with customizable workflows. BMC Bioinformatics 12, 277 (2011).

33. Sharov, A. A. \& Ko, M. S. Exhaustive search for over-represented DNA sequence motifs with CisFinder. DNA Res. 16, 261-273 (2009).

34. Ji, H. et al. An integrated software system for analyzing ChIP-chip and ChIP-seq data. Nature Biotechnol. 26, 1293-1300 (2008).

35. Mortazavi, A., Williams, B. A., McCue, K., Schaeffer, L. \& Wold, B. Mapping and quantifying mammalian transcriptomes by RNA-Seq. Nature Methods $\mathbf{5}$, 621-628 (2008).

36. Jacobs, S. A., Fischle, W. \& Khorasanizadeh, S. Assays for the determination of structure and dynamics of the interaction of the chromodomain with histone peptides. Methods Enzymol. 376, 131-148 (2004).

37. Zuber, J. et al. Mouse models of human AML accurately predict chemotherapy response. Genes Dev. 23, 877-889 (2009). 